\title{
Evaluation of current treatment technology for black-odor rivers
}

\author{
Chen Song ${ }^{1,2,3, a}$, Xiaoling Liu ${ }^{2,3, b}$, Yonghui Song ${ }^{1,2,3, \mathrm{c}^{\star}}$, Ruixia Liu ${ }^{2,3, \mathrm{~d}}$, Jianfeng \\ Peng ${ }^{2,3, \mathrm{e}}$
}

${ }^{1}$ College of Water Science, Beijing Normal University, Beijing, 100018, China

${ }^{2}$ State Key Laboratory of Environmental Criteria and Risk Assessment, Chinese Research Academy of Environmental Sciences, Beijing, 100012, China

${ }^{3}$ Department of Urban Water Environmental Research, Chinese Research Academy of Environmental Sciences, Beijing, 100012, China

"Corresponding author

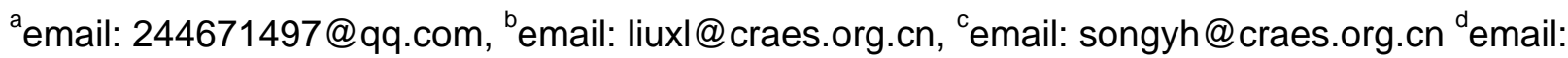
liurx@craes.org.cn, ${ }^{e} e m a i l:$ pengjf@craes.org.cn

Keywords: black-odor, urban rivers, treatment, techniques

Abstract. A large number of urban rivers in China have been heavily polluted, and occurred so-called black-odor appearance. How to treat and dispose these black-odor urban rivers has become an urgent and important issue for Chinese government. In the last few decades, the treatment techniques for heavily polluted rivers have attracted more and more attentions. Compared with other heavily polluted rivers, the black-odor river has its typical characteristics, like odor-smelling. Therefore, not all earlier developed techniques are suitable for the treatment of the black-odor rivers. This paper provided a review of the literature on the main techniques applied to the pollutants removal for the black-odor rivers including physical, chemical and biological methods. The advantages and disadvantages of these techniques were also evaluated. The most promising techniques were highlighted, and some suggestions for the treatment of the black-odor rivers were also given.

\section{Introduction}

The phenomenon of urban river water becoming black-odor commonly occurred in China. For example, the total length of the black-odor rivers is $12,000 \mathrm{~km}$ in Zhejiang province, which accounted for $10 \%$ of the whole rivers in this province. In 18 cities of Henan province, there are 34 black-odor rivers that are 56.7 percent of the total amount of the rivers in this province. In Guangzhou, 135 rivers have presented black-odor phenomenon, while 413 rivers for Chengdu. This phenomena is mainly resulted from the deterioration of ecological environmental, and seriously affect people's normal life. It has becoming one of the vital water environment problems.

Before 1990s, the issue of environmental protection was not paid more attentions in China with the reforming and opening, Chinese economy has entered a stage of rapid development. Because of the uneven development of economic and technological, policy difference, and management loopholes, a large amount of untreated industrial and domestic wastewater were directly discharged into rivers, resulting in the frequent heavy pollution. Since the middle 1990s, the increasingly serious pollution of the rivers has widely aroused the concern of the government and the people in China. However, the conservation and management of rivers still followed the traditional concept of urban planning and management due to the technical limitation and lack of experience. The aims of most techniques still focused on the water pollution control and water conservancy project. Many rivers remained black-odor year by year. In China, more than $80 \%$ of urban rivers suffer heavily polluted. Many of them even appear seasonal and perennial black-odor phenomenon.

\section{The technologies for the treatment of black-odor rivers}

Rivers are important carriers of urban civilization and development, especially the multi-function urban rivers that have become new spiritual and cultural needs, vital part of ecological civilization and 
harmonious society as well as city identity. However, the rapid development of global industrialization and urbanization aggravates the river pollution constantly. Many rivers have been polluted heavily, and have even become black-odor. Although the studies on the technology for black-odor rivers treatment in China are later than that in other developed countries, the developing stages of technology are similar. The techniques have experienced three stages including water conservancy project, environmental protection and comprehensive management as well as ecological restoration.

Table 1 Evaluation of conventional technical for the treatment of black-odor rivers

\begin{tabular}{ccc}
\hline Methods & Representative technology & Application \\
Physical methods & Sediment dredging, water diversion, & Often used as pretreatment process \\
and aeration & Often used as pretreatment process \\
Chemical methods & Fenton reagent and ozone & and emergency control measures \\
Biological methods & Microbiological, phytoremediation, & Often used as advanced treatment \\
& and biological manipulation process & and long term maintenance \\
\hline
\end{tabular}

Physical methods. The obvious advantages of physical methods are rapid and effective. The common methods include sediment dredging, water diversion and aeration. These methods are widely used to pretreat rivers. However, the water qualities of rivers are improved temporarily using these physical methods with the huge quantity of project and investment. Sediment dredging can greatly reduce the pollution from river sediment to the overlying water, thereby improving the water quality and dredging the river. As early as 1960s, the method had been widely studied and applied to remove the pollutants in sediment and improve water storage and shipping capacity $[1,2]$. Nevertheless, river sediment can't be completely removed by this method due to the limitations of engineering conditions and natural factors. Meanwhile, sediment dredging method brings out benthic organisms and microorganisms, which broke the long-term ecological balance. Furthermore, the brought sediment needs to be further treated in order to avoid the secondary pollution. This project of sediment dredging demands sufficient funds to maintain, especially the large area dredging project. In the method of water diversion, clean rivers is used to dilute black-odor rivers, and then to improve the self-purification ability of black-odor river. This method is one of the common and effective auxiliary ways that improve water quality. But this method requires extreme geographical conditions with huge amounts of water resources, and also may cause adjacent rivers pollution. Because of less investment, quick effect, no secondary pollution, the aeration method is adopted as a conventional river treatment technology in the United States, Germany and other developed countries since 1950s [3]. This method can make up for the limited ability of the atmospheric complex oxygen, and therefore improve the mass transfer and diffusion of oxygen in the rivers. The dissolved oxygen (DO) into the rivers can be used to oxidize and restore the blacking pollutants, inhibit anaerobic microbial growth and enhance aerobic microbial activity, thus reducing pollutant load in the rivers and improving the ecological environment. However, higher demands of facility and energy-consumption limit its application, especially in energy shortages and remote areas.

Chemical methods. The refractory organics under specific reaction conditions can be mineralized or oxidized by chemical methods. These method also have great advantages on the removal of environmental estrogens and are usually used as pretreatment process and emergency control measures because of high cost of reagent and energy. Coagulation method is used to treat sewage and rivers as early as 1990s because it could not only reduce turbidity, chroma and smell, but also remove many toxic and harmful pollutants, especially sulfide that is the main blackening and stinking pollutants [4]. Using coagulation to treat black-odor rivers has been proven to be feasible. Several new methods are derived from the coagulation method like chemical coagulation and electro coagulation. These two methods are the two main coagulation technology. However, only chemical coagulation is adopted in 
most of practical operation. The scale and product application of electric flocculation are still limited due to the high energy cost and electrode passivation. Fenton reagent, as a new AOP, was used widely to improve the clarity and remove volatile organic compounds (VOCs) since the early 21 st century. The results indicated that the water quality could be enhanced by Fenton reagent method, and the chroma and turbidity decreased by $73.73 \%$ and $93.11 \%$, respectively [5]. Both of photo-Fenton and electro-Fenton are the two main developing direction for the Fenton method. Some studies showed that photo-Fenton was an effective environment friendly technology, which could remove VOCs and inhibit the discharge of by-product during the process. Similarly with the electro coagulation method, the further development and application of electro-Fenton method was also restricted due to the low current efficiency and instability of electrode material. Since the first ozone experimental device disinfected for tap water was designed in 1908 in France, the ozone technology was applied to treat municipal sewage and industrial wastewater. Until 1970s, combined with activated carbon or other technologies, the ozone technology was regarded as the main advanced water treatment method that was usually adopted to remove the chemical pollutants of drinking water. This method has the advantages of disinfection, decolorization and smell control [6]. Although the ozone technology has the high efficiency in disinfection, decolorization, and smell control, some new VOCs may be generated during this process.

Biological methods. Compare with the physical and chemical methods, the biological methods are more economical and environmentally friendly, and have been widely used for the field of water treatment. The water quality of a black-odor river can be improved by microbial consortium, especially complex microbial community. The removal ratio of $\mathrm{COD}, \mathrm{NH}_{3}-\mathrm{N}$, odor level and chroma in the black-odor river separately reached $54.4 \%, 11.65 \%, 91.65 \%$ and $89.6 \%$ using the complex microbial community that consisted of Bacillus sp. NS1, Aeromonas hydrophila B23 and Oedogonium Link.sp. [7]. The phytoremediation methods mainly include pollutants absorption, extraction, decomposition, transformation and immobilization. Nymphaea that grows in the black-odor river could be used to remove $\mathrm{COD}, \mathrm{TN}, \mathrm{NH}_{3}-\mathrm{N}, \mathrm{TP}$ and dissolved phosphorus, and their removal ratios reach above $60 \%$ [8]. Phytoremediation often plays an important role in several aspects including the maintenance of the traditional functions of water conservancy projects, the protection of river ecosystem, the improvement of the living environment and the strengthen of cultural landscape. Biological manipulation process was proposed in 1975 by Shapiro. The essence is to reduce the production of phytoplankton biomass by food chain in order to avoid eutrophication or control algae biomass. Previous research indicated that phytoplankton biomass showed negatively correlation with plant zooplankton biomass, but positively correlated with number of carnivorous zooplankton and concentration of $\mathrm{NH}_{3}-\mathrm{N}$ and partial phosphate salt [9]. Although this process had significant effect on improving water quality and ecological environment, some survey work are also necessary in order to avoid species invasion, food chain destruction and other problems.

\section{Suggestions}

With the development of treatment technology, many black-odor rivers in China have become clean again such as Suzhou River, Qinhuai River and Jimo River, and so on. But there are still many inadequacies and many aspects needed to be further studied.

In-situ remediation process is the only way for river treatment in China no matter the physical, chemical and biological methods in current projects. This process may cause some impact on river ecology, especially the environmental safety of the biological methods. Generally, for in-suit remediation processes, a large scale civil engineering or water conservancy project is needed to be constructed. This certainly causes some negative impact on the traffic nearby, especially in the large cities of China such as Beijing, Shanghai, and Nanjing. Furthermore, the amount and cost of chemical or biological reagents for water treatment must be numerous because of the regular spray of the reagents. Ex-suit remediation process may be a new choice for rivers treatment due to the lowered cost of chemical or biological reagent used in a limited treatment area in comparison with the in-situ 
process. However, re-utilization and treatment efficiency of ex-suit remediation process still limits its practical application. How to achieve re-utilization and improve treatment efficiency are the main tasks in the future. In addition, $\mathrm{COD}, \mathrm{NH}_{3}-\mathrm{N}, \mathrm{TP}$ and other indicators are often monitored to evaluate the effect on rivers treatment in practice, but the balance of river ecology is ignored. Although water quality reaches the national standard in a short term, the rivers still appear seasonal black-odor phenomenon.

Facing the increasingly severe water environmental problems, only relying on treatment technology cannot solve the phenomenon of black-odor in urban rivers. The policy, law and financial aid system is needed, such as improving the comprehensive management capabilities, increasing investment in environmental protection, promoting the development of environmental protection industry, and encouraging new technology researches.

\section{Acknowledgements}

This work was financially supported by the Major Science and Technology Program for Water Pollution Control and Treatment of China (2012ZX07202-005), and the National Natural Science Foundation of China (No.21277133).

\section{References}

[1] B.R. Moore: Water Research Vol. 3 (1969), p. 777-778.

[2] D.J. Grimes: Applied Microbiology Vol. 29 (1975), p. 109-111.

[3] K.R. Imhoff and D. Albrecht: Journal (Water Pollution Control Federation) Vol. 49 (1977), p. 1959-1967.

[4] H.L. Sheng and M.L. Chi: Water Research Vol. 27 (1993), p. 1743-1748.

[5] Z.Y. Chen, G.X. Wang, F. Yang, W.W. Xu, L. Wang and Z.F. Yu: Chinese Journal of Environmental Engineering Vol. 6 (2012), p. 1591-1594.

[6] T. Syuji: Ozone: Science and Engineering Vol. 13 (1991), p. 623-637.

[7] F.F. Huang: Central China Normal University Wuhan (2012).

[8] X.M. Lu, P.Z. Lu and J.J. Chen: Toxicological and Environmental Chemistry Vol. 94 (2012), p. $1-12$

[9] K. Anna, G. Ryszard and D. Renata: Plos One (2015). 